\title{
Propiedades de sistemas tipo M/G/1/K con estaciones en serie
}

\author{
Properties of Multi-Stage $M / G / 1 / K$ Queues with Series Arrangement \\ Hernández-González Salvador \\ Departamento de Ingeniería Industrial \\ Instituto Tecnológico de Celaya \\ Correo:salvador.hernandez@itcelaya.edu.mx \\ Hernández-Ripalda Manuel Darío \\ Departamento de Ingeniería Industrial \\ Instituto Tecnológico de Celaya \\ Correo:dario.hernandez@itcelaya.edu.mx \\ Flores-De la Mota Idalia \\ División de Ingeniería Mecánica e Industrial \\ Facultad de Ingeniería \\ Universidad Nacional Autónoma de México \\ Correo:idalia@unam.mx \\ Vázquez-López José Antonio \\ Departamento de Ingeniería Industrial \\ Instituto Tecnológico de Celaya \\ Correo: antonio.vazquez@itcelaya.edu.mx
}

Información del artículo: recibido: diciembre de 2012, aceptado: junio de 2014

\section{Resumen}

En este trabajo se presenta una adecuación al método de Buzacott y Shanthikumar (1993) para calcular analíticamente las propiedades de sistemas de líneas de espera tipo $M / G / 1 / K$ y arreglo en serie. Se realizaron pruebas de validación empleando simulación y se observó que, con la modificación propuesta, el método tiene un grado de exactitud aceptable para obtener las propiedades de sistemas $M / G / 1 / K$ en serie, con lo que se puede considerar como una alternativa a otro método propuesto en la literatura para dicha clase de sistemas. Al ser un procedimiento que requiere menos cálculos, es de gran utilidad para responsables en la toma de decisiones, que requieren implementar herramientas para analizar el desempeño de sistemas de líneas de espera en ambientes de producción y servicios.

\footnotetext{
Abstract

We propose a modification to the Buzacott $\mathcal{E}$ Shantikumar (1993) method to evaluate the properties of $M / G / 1 / K$ queues with series arrangement. We made several tests and compared with simulation to validate the results. We observed that, with the proposed modification, the method has an acceptable level of exactitude. It can be considered an alternative calculation to other methods in the literature. The fact that this method requires less computational effort, can be useful to practitioners and managers that require tools to analyze the performance of systems in production and services areas.
}

\section{Descriptores:}

- líneas de espera

- análisis

- descomposición

- buffer

- estaciones múltiples

- arreglo en serie

\section{Keywords:}

- queue

- decomposition

- buffer

- multistation

- series 


\section{Introducción}

Aunque no siempre evidente, las filas o líneas de espera que se forman ante una estación o servidor son parte de las propiedades de cualquier sistema, sea de producción, manufactura o de servicios. En la administración de un sistema, es frecuente tomar decisiones tomando como base la perspectiva de reducir en lo posible los costos asociados con la espera por parte de los clientes.

El estudio de las líneas de espera se relaciona con la cuantificación del fenómeno de esperar a ser atendido, por medidas de desempeño representativas, tales como el número promedio de clientes esperando ser atendidos, tiempo promedio de espera en la fila o el uso promedio de la instalación.

\section{Problema}

El estudio y análisis de los sistemas de líneas de espera requiere métodos y modelos para calcular sus propiedades con un alto grado de exactitud; de especial interés son los sistemas con estaciones en serie y capacidad limitada en la línea de espera (comúnmente llamado buffer). Esta clase de sistemas son comunes en prácticamente cualquier ámbito, por ejemplo, en los sistemas de manufactura y producción existe por lo general un espacio destinado al almacenamiento de piezas o lotes que serán procesados en los equipos, este inventario se mantiene para que en caso de que una estación detenga su producción, la siguiente estación en la línea no deba parar por falta de suministro. Si el buffer de una máquina se encuentra lleno, entonces la estación anterior detiene su producción hasta que exista espacio suficiente para recibir al menos una unidad de producción (Buzacott y Shanthikumar, 1993).

$\mathrm{Si}$ se suponen tiempos exponenciales, o bien tipo Erlang, entonces se pueden utilizar cadenas de Markov para obtener los valores exactos de las propiedades de estos sistemas, sin embargo el número de estados crece de manera explosiva y por lo tanto las cadenas son poco prácticas. Otro medio son las aproximaciones por ecuaciones y modelos analíticos.

Para el caso en donde el tiempo de servicio en la estación es de tipo Markoviano existen modelos analíticos conocidos, sin embargo, cuando el tiempo de servicio de las estaciones es no-Markoviano (M/G/1/K según la notación Kendall), el cálculo de las propiedades se dificulta por la carencia (al menos hasta hace algunos años) de modelos analíticos y expresiones para realizar el análisis de dicha clase de sistemas. Para su estudio, lo común era recurrir a modelos de simulación para caracterizar y analizar dicha clase de sistemas, dada la carencia de modelos analíticos para aproximar sus propiedades. Con esta perspectiva, este trabajo tiene como objetivos:

1. Presentar una adecuación a un método conocido, que permite calcular analíticamente las propiedades de sistemas $M / G / 1 / K$ en serie.

2. Validar y cuantificar el grado de exactitud del método con las adecuaciones propuestas.

\section{Antecedentes}

Los métodos desarrollados para el cálculo de las propiedades de sistemas de líneas de espera $M / G / 1 / K$ con varias etapas o estaciones dispuestas en serie, se pueden encontrar en: Hillier y Boling (1967); Takahashi et al. (1980) en donde se utiliza el enfoque de Jackson de redes de líneas de espera; en Perros y Altiok (1986) y Gershwin (1987), donde se utilizan cadenas de Markov, en Kerbache y MacGregor (1987); en MacGregor y Daskalaki (1988) en donde proponen el método de expansión generalizada (GEM); en Tan y Yeralan (1997) que emplean programación lineal, y Van et al. (2005) donde emplean descomposición. Finalmente, en los libros de Buzacott y Shanthikumar (1993); Altiok (1996); Curry y Feldman (2011) se pueden encontrar los respectivos procedimientos.

En los trabajos mencionados los supuestos son: un solo servidor, llegadas de clientes tipo Poisson, y tiempos de servicio exponenciales para realizar el análisis. Para el caso de los tiempos de servicio generales $(G)$ solo se reportaron casos resueltos muy pequeños como por ejemplo en Pollock et al. (1985), esto se debe a que aún no se obtenían ecuaciones que fueran fáciles de manipular y que permitieran aproximar el número de clientes en el sistema, probabilidad de bloqueo y tiempo ciclo de esta clase de sistemas; en consecuencia se debía recurrir a modelos de simulación para determinar estas propiedades (tabla 1 para la notación y la relación de símbolos empleados).

En el trabajo de Gelenbe (1975) se reporta una ecuación para aproximar el tamaño de una línea de espera con buffer frente a una estación y tiempos de servicio no-Markovianos, sin embargo es poco práctica como medio analítico; en realidad es con el trabajo de Kimura (1996) donde hay un gran avance, ya que se desarrolla una ecuación para calcular el tamaño del buffer en un sistema con servicio general, la cual es muy práctica. El siguiente paso lo da MacGregor en 2004; retoma la propuesta de Kimura y desarrolla las ecuaciones respectivas para aproximar el número de clientes en el sistema, la probabilidad de bloqueo y la probabilidad de que la 
estación se encuentre desocupada, para el caso en donde los tiempos de servicio son no Markovianos.

Es importante resaltar que las ecuaciones referidas en el párrafo anterior requieren como parámetros la congestión del sistema ( $\rho)$, el tamaño del buffer $(K)$ y el coeficiente de variación cuadrático del servicio de la estación $\left(C V_{T_{S}}^{2}\right)$ (tabla 1$)$, en donde este último es una medida de la variación del servicio que proporciona una estación; se calcula como sigue:

$$
C V_{T_{S}}^{2}=\frac{\sigma_{T_{S}}^{2}}{\left(E\left[T_{S}\right]\right)^{2}}
$$

El coeficiente de variación cuadrático del servicio es la relación de la varianza del tiempo de servicio $\left(\sigma_{T}^{2}\right)$ y el cuadrado del valor esperado del tiempo de servicio $\left(\mathrm{E}\left[T_{S}\right]\right)^{2}$.

Con los resultados de MacGregor fue posible extender el GEM a los sistemas $M / G / 1 / K$ y $M / G / c / K$ con estaciones en serie y realizar el cálculo y caracterización de sus propiedades. En la serie de trabajos de Cruz y MacGregor (2007); Cruz et al. (2008) y Van et al. (2010) se han reportado de manera muy completa las características

Tabla 1. Notación y símbolos utilizados

\begin{tabular}{ll}
\hline$K$ & $\begin{array}{l}\text { Espacio de almacenamiento disponible fuente a la estación } \\
(\text { Buffer })\end{array}$ \\
$k$ & Parámetro de forma de la función de distribución gamma \\
$E\left[T_{S}\right]$ & Valor esperado del tiempo de servicio de la estación \\
$L_{q}$ & Número de clientes promedio formados frente a la estación \\
$L_{S}$ & Número de clientes promedio en el sistema índice de estación \\
$i$ & Índice de estación \\
$m$ & Número total de estaciones o máquinas \\
$p_{K}$ & Probabilidad de bloqueo de la estación (Búffer lleno) \\
$p_{0}$ & Probabilidad de que la estación se encuentre vacía \\
$M$ & Sistema markoviano \\
$G$ & Sistema no Markoviano o general \\
$e$ & Número natural = 2.7182 \\
$c$ & Número de servidores \\
$\lambda$ & Tasa de llegadas \\
$\mu$ & tasa de servicio \\
$\rho$ & Congestión del sistema, $\lambda / \mu$ \\
$\sigma_{T_{S}}^{2}$ & Varianza del tiempo de servicio \\
$\mu_{i d}$ & Tasa efectiva de salida dela estación $i$ \\
$\theta$ & Parámetro de escala de la función de distribución gamma \\
$\bar{\lambda}_{i}$ & Tasa efectiva de llegadas a la estación $i$ \\
$C V_{T_{S}}^{2}=\frac{\sigma_{T_{S}}^{2}}{\left(E\left[T_{S}\right]\right)^{2}}$ & Coeficiente de la variiación cuadrático del tiempo de \\
\hline
\end{tabular}

de sistemas $M / G / 1 / K$ y $M / G / c / K$; cabe resaltar que esto ha permitido desarrollar métodos heurísticos para la solución analítica de otro problema conocido como problema de localización del buffer (BAP).

El método de expansión generalizada (GEM) es un procedimiento de descomposición que consta de tres fases:

1. Reconfiguración de la red de estaciones.

2. Estimación de parámetros.

3. Eliminación y actualización hacia atrás.

Se trata de un método en donde el paso más importante es la reconfiguración, ya que el sistema original se modifica agregando una estación o nodo ficticio antes de cada estación, el cual sirve para modelar el bloqueo de dicha estación. Una vez realizada la expansión, el siguiente paso es calcular las propiedades de cada estación o nodo (incluyendo los ficticios).

Un inconveniente del método GEM es la adición de nodos nuevos, lo que aumenta la cantidad de cálculos. Se recomienda consultar Kerbache y MacGregor (1987); MacGregor y Daskalaki (1988) para una descripción completa de dicho método.

La aportación de este trabajo es mostrar el cálculo analítico de las propiedades de sistemas $M / G / 1 / K$ en serie, empleando el método de Buzacott y Shanthikumar (1993) y las ecuaciones reportadas en MacGregor; usando simulación se verificó el grado de exactitud, el cual resultó ser aceptable; el método de Buzacott y Shanthikumar tiene la ventaja de que requiere menos cálculos (no es necesario agregar nodos nuevos para modelar el bloqueo) y es viable su implementación como plantilla de hoja de cálculo, lo cual en términos prácticos es muy importante en ambientes sumamente dinámicos que es lo común en la vida real.

Fórmulas para el cálculo de $\mathrm{p}_{0}, \mathrm{p}_{\mathrm{K}} \mathrm{y} \mathrm{L}_{\mathrm{S}}$ en sistemas $\mathrm{M} / \mathrm{G} / 1 / \mathrm{K}$

Como se comentó, Kimura propuso en 1996 una ecuación para calcular el tamaño del buffer para sistemas con tiempos de servicio generales, MacGregor (2004) retoma y modifica esta fórmula de manera empírica; además ahí se proponen las ecuaciones para aproximar los valo- 
res de la probabilidad de bloqueo de la estación y el número promedio de clientes en el sistema. Para el caso de la probabilidad de bloqueo $p_{K}$ la ecuación es la siguiente:

$p_{K}=\frac{\rho^{\beta_{1}}(1-\rho)}{\rho^{2 \beta_{2}}-1}$

donde los términos $\beta_{1}$ y $\beta_{2}$ tienen las siguientes expresiones

$$
\begin{aligned}
& \beta_{1}=\left(\sqrt{\rho} C V_{T_{S}}^{2}-\sqrt{\rho}+2 K\right) /\left(2+\sqrt{\rho} C V_{T_{S}}^{2}-\sqrt{\rho}\right) \\
& \beta_{2}=\left(1+\sqrt{\rho} C V_{T_{S}}^{2}-\sqrt{\rho}+K\right) /\left(2+\sqrt{\rho} C V_{T_{S}}^{2}-\sqrt{\rho}\right)
\end{aligned}
$$

La probabilidad de que el sistema se encuentre vacío $p_{0}$ se calcula con la siguiente expresión

$$
p_{0}=\frac{(\rho-1)}{\rho^{2 \beta_{2}}-1}
$$

donde (2c) corresponde al término $\beta_{2}$. Se debe señalar que, como información, se requieren el cuadrado del coeficiente de variación del servicio y la congestión del sistema (tabla 1). Para el caso del número de clientes en el sistema $\left(L_{S}\right)$, ver la ecuación 4 .

En este caso, es necesario contar con el parámetro de congestión del sistema $(\rho)$, la probabilidad de bloqueo $\left(p_{K}\right)$ y el coeficiente de variación cuadrático del servicio de la estación $\left(C V_{T_{S}}^{2}\right)$.

\section{Método de descomposición para sistemas en serie}

A continuación se muestra el método de descomposición propuesto por Buzacott y Shanthikumar (1993) para calcular las propiedades de sistemas de $M / M / 1 / K$ en serie. El método calcula de manera recursiva las pro- piedades de un sistema formado por las estaciones $m$, $m-1, \ldots, 2,1$. La máquina $m$ nunca está bloqueada y la máquina 1 nunca sufre de escasez de material (no requiere buffer). El procedimiento es el siguiente

Paso 1. Fijar $\mu_{m d}=\mu_{m}$

Paso 2. Para $i=m, \ldots, 2$, hacer

Si $\mu_{i d}<\lambda$ entonces el sistema es inestable, terminar

En otro caso, encontrar $\bar{\lambda}_{i}$ resolviendo

$$
\lambda_{i}\left(1-p_{K}\left(\bar{\lambda}_{1}, \mu_{i d}, K_{i}+1\right)\right)=\lambda
$$

Establecer

$$
\begin{aligned}
& \frac{1}{\mu_{i-1, d}}=\frac{1}{\mu_{i-1}}+\left(\frac{1}{\mu_{i, d}}\right) p_{K}\left(\bar{\lambda}_{i}, \mu_{i, d}, K_{i}\right) \\
& L_{i}=\bar{L}_{M / M / 1 / K}\left(\bar{\lambda}_{i}, \mu_{i, d}, K_{i}+1\right)
\end{aligned}
$$

Paso 3. Calcular

$$
L_{1}=\bar{L}_{M / M / 1 / K}\left(\lambda, \mu_{i, d}\right)
$$

\section{Paso 4. Terminar}

En el primer paso, la tasa efectiva de salida de la última estación es igual a su tasa nominal de servicio. En el paso 2 se verifica que la estación $i$ tenga capacidad para atender la demanda, si esta condición se cumple entonces se calcula la probabilidad de que la estación se encuentre bloqueada $\left(\mathrm{p}_{\mathrm{K}}\right)$ y a continuación se resuelve la ecuación no lineal (5) para obtener la tasa efectiva de llegadas a la estación $\left(\lambda_{\mathrm{i}}\right)$; para tal efecto puede utilizarse algún método de búsqueda como Regula-falsi. En el mismo paso 2, se debe calcular el tiempo de salida efectivo (6) y a continuación el número de clientes en el sis-

$L_{S}= \begin{cases}\frac{\rho\left(\ln (1 / \rho)+p_{K} \ln \left(p_{K} /\left(1-\rho+\rho p_{K}\right)\right)+p_{k} \ln (\rho)\right)\left(2+\sqrt{\rho / e^{C V^{2}}} C V_{T_{S}}^{2}-\sqrt{\rho / e^{C V^{2}}}\right)}{2 \ln (\rho)(\rho-1)} & \text { si } C V_{T_{S}}{ }^{2} \leq 1 \\ \frac{\rho\left(\ln (1 / \rho)+p_{K} \ln \left(p_{k} /\left(1-\rho+\rho p_{K}\right)\right)+p_{k} \ln (\rho)\right)\left(2+\sqrt{\rho / e} C V_{T_{S}}^{2}-\sqrt{\rho / e}\right)}{2 \ln (\rho)(\rho-1)} & \text { si } C V_{T_{S}}{ }^{2}>1\end{cases}$ 
tema (7). Estos cálculos se inician en la máquina $m$ y se repiten hasta la máquina 2. En el paso 3 se determina el número de clientes en el sistema de la máquina 1 con (8), deteniendo el método en el paso 4.

Como se mencionó, el método se desarrolló para sistemas donde la tasa de servicio es de tipo exponencial y en esencia se resuelven de manera recursiva los balances de flujo de entrada-salida hacia cada estación o nodo.

Para el caso de sistemas $M / G / 1 / K$ en serie, se pueden sustituir las ecuaciones propuestas por MacGregor: para calcular la probabilidad de bloqueo del sistema se debe utilizar (2a, b y c), la probabilidad de que el sistema esté vacío se calcula con (3) y para el cálculo del número de clientes en el sistema se debe emplear (4), el resto del método no requiere modificaciones, a continuación se muestran las pruebas y la validación correspondiente.

\section{Experimentos y resultados}

Se realizó un conjunto de pruebas para verificar el desempeño del método en el cálculo de las propiedades de sistemas tipo $M / G / 1$ en serie, con distintos escenarios del coeficiente de variación cuadrático que es el parámetro que incorpora la variación del sistema y permite aproximar aquellos sistemas con servicios que no son de tipo exponencial; para tal efecto se utilizaron las ecuaciones de MacGregor. Se resalta el hecho de que dicho método de Buzacott en su forma original supone que $C V_{T_{S}}^{2}=1$.

Las ecuaciones se programaron en una plantilla de hoja de cálculo; por el momento solo se muestra la imagen del arreglo de dicha plantilla para efectos ilustrativos, dado que el objetivo principal de este reporte es estudiar la efectividad de las ecuaciones para predecir las propiedades, así como realizar algunas conjeturas sobre el efecto de la variación (medida a través de $C V_{T_{S}}^{2}$ ) sobre las propiedades del sistema (figura 1).

Las pruebas se dividieron en dos partes. En la primera serie se considera que todas las estaciones tienen el mismo tiempo de servicio, en la segunda serie de pruebas, se consideró que la estación central es el cuello de botella del sistema; los resultados obtenidos de manera analítica se validaron mediante simulación.

\section{Servicio uniforme}

En la tabla 2 se muestran los datos empleados para las pruebas en donde la tasa de servicio y el $C V_{T_{\mathrm{S}}}^{2}$ son iguales en todas las estaciones. En la columna 2 se muestra el coeficiente de variación, en las columnas 3 y 4 se dan los datos del parámetro de escala y parámetro de forma de la función de distribución gamma, con estos datos se obtienen el valor esperado y la varianza del tiempo de servicio, los cuales se muestran en las columnas 5 y 6 .

A modo de ejemplo se describe la prueba 1: a la función gamma con $k=1$ y $\theta=0.65$, le corresponde un tiempo de servicio con valor esperado de $0.65 \mathrm{~h}$ y varianza de $0.4225 \mathrm{~h}^{2}$ y se puede verificar que corresponde al caso donde el tiempo de servicio es de tipo exponencial; los parámetros del valor esperado y coeficiente de variación son los requeridos para realizar el cálculo analítico.

Para validar los resultados obtenidos con el método analítico se construyó el modelo respectivo de simula-

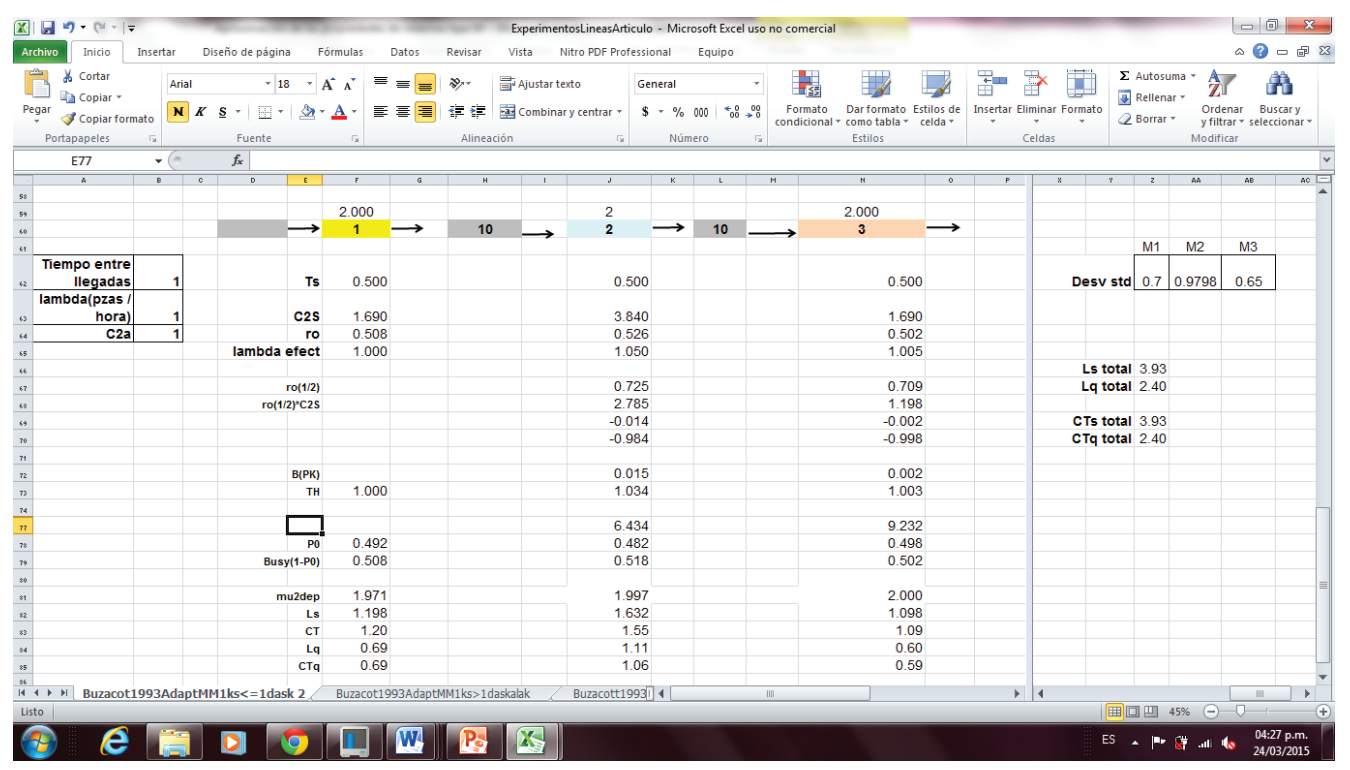

Figura 1. Plantilla para análisis de sistemas $\mathrm{M} / \mathrm{G} / 1 / \mathrm{K}$ con estaciones en serie 
ción en el paquete Arena (Altiok y Melamed 2007), se utilizó una tasa de llegadas $\lambda=1$ de tipo exponencial; en el mismo modelo de simulación se utilizó la función de distribución gamma para los tiempos de servicio utilizando los datos de la columna 3 para el parámetro de forma y de la columna 4 para el parámetro de escala (tabla 2). Los parámetros de tamaño de buffer de cada estación, el número de horas de simulación y el número de corridas de simulación realizadas se muestran en la tabla 3.

En la figura 2 se muestran de manera simultánea el número de clientes $\left(L_{S}\right)$ obtenido de manera analítica y con el modelo de simulación; se observa que a medida que existe mayor variación en el servicio $\left(C V_{T_{S}}^{2}>1\right)$, el número de clientes promedio en el sistema se incrementa. Lo anterior es acorde con lo que sucedería en la realidad: una mayor variación en todas las estaciones puede implicar en una operación real que, de cliente a cliente, existen diferencias considerables en el servicio; aquí las causas son diversas y el parámetro que proporciona la información de esas variaciones es el coeficiente de variación cuadrático.
En cuanto a la exactitud de las ecuaciones se puede observar en la tabla 4 que cuando $C V_{T_{S}}^{2}=1.25$ y 1.5 la diferencia del resultado analítico con respecto al simulado es -6.7 y $-11.9 \%$ respectivamente, en las pruebas $C V_{T_{\mathrm{S}}}^{2}=1,0.85$ y 0.75 , las diferencias son $-0.9,3.6$ y $4.3 \%$ respecto al resultado que se obtiene mediante simulación (figura 3, tabla 4).

En cuanto a la probabilidad de bloqueo $\left(p_{K}\right)$ se aprecia que a medida que $C V_{T_{S}}^{2}$ aumenta, entonces la probabilidad de que una estación se encuentre bloqueada se incrementa también (tabla 5).

Se observa también que para valores de $C V_{T_{S}}^{2}=1.25$ y 1.5 el modelo analítico aproxima bien el valor de $p_{K^{\prime}}$ en cambio, cuando $C V_{T_{\mathrm{s}}}^{2}=1,0.85$ y 0.75 , el resultado obtenido de manera analítica es mayor que el resultado obtenido con simulación.

Por último, en cuanto a la probabilidad de que la estación se encuentre vacía $\left(p_{0}\right)$, en todos los casos el modelo analítico obtiene el mismo valor que se obtiene mediante simulación.

Tabla 2. Parámetros de la función gamma, valor esperado y varianza de las 3 estaciones

\begin{tabular}{cccccc}
\hline & & \multicolumn{2}{c}{$\begin{array}{c}\text { Parámetros de la función } \\
\text { gamma para el modelo de } \\
\text { simulación }\end{array}$} & \multicolumn{2}{c}{$\begin{array}{c}\text { Datos para el modelo } \\
\text { analítico }\end{array}$} \\
\hline Prueba & $C V_{T_{s}}^{2}$ & $\begin{array}{c}\text { Parámetro de } \\
\text { forma }(k)\end{array}$ & $\begin{array}{c}\text { Parámetro de } \\
\text { escala }(\theta)\end{array}$ & $\begin{array}{c}\text { Valor esperado } \\
\text { del tiempo de } \\
\text { servicio } k \times \theta\end{array}$ & $\begin{array}{c}\text { Varianza } \\
k \times \theta^{2}\end{array}$ \\
\hline 1 & 1 & 1 & 0.65 & 0.65 & 0.4225 \\
2 & 1.25 & 0.8 & 0.8125 & 0.65 & 0.5281 \\
3 & 1.5 & 0.6666 & 0.97509 & 0.65 & 0.6338 \\
4 & 0.85 & 1.1764 & 0.5525 & 0.65 & 0.35914 \\
5 & 0.75 & 1.3333 & 0.48750 & 0.65 & 0.31687 \\
\hline
\end{tabular}

Tabla 3. Datos adicionales de las pruebas

\begin{tabular}{lc}
\hline \multicolumn{1}{c}{ Parámetro } & Valor \\
\hline Número de estaciones en serie & 3 \\
Tamaño del buffer & $(-, 10,10)$ \\
Tasa de llegadas $(\lambda)$ & 1 \\
Horas de simulación & 100,000 \\
Corridas de simulación & 3 \\
\hline
\end{tabular}

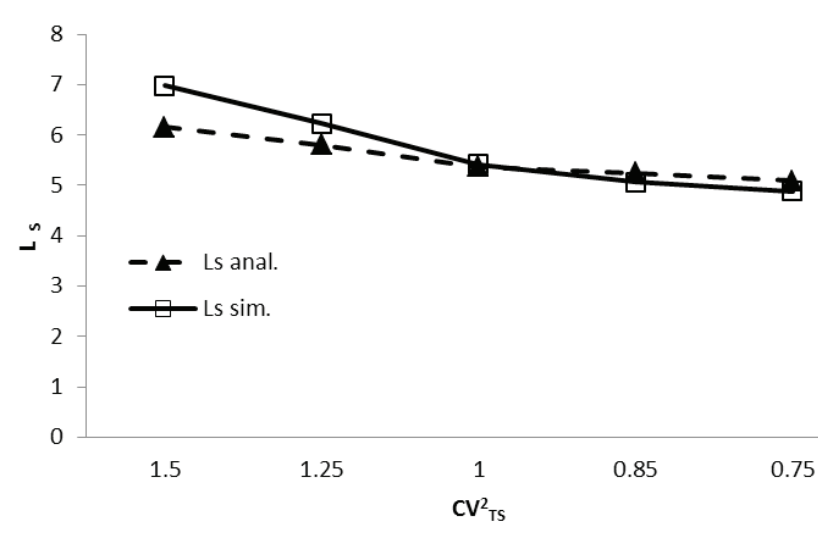

Figura 2. Número de clientes en el sistema vs $C V_{T_{S}}^{2}$ 


\section{Cuello de botella}

En la segunda serie de experimentos se estudió la capacidad del método analítico para predecir las propiedades de sistemas que tienen cuello de botella. El cuello de botella se define como la máquina o estación más lenta (Lawrence \& Buss, 1996) y en esta serie de pruebas se colocó en la máquina del centro. Para las máquinas 1 y 3 se mantuvo en todas las pruebas un tiempo de servicio de $0.65 \mathrm{~h}$ de tipo exponencial $\left(C V_{T_{\mathrm{S}}}^{2}=1\right)$. En el caso del cuello de botella, se experimentó incrementando a $0.8 \mathrm{~h}$ el tiempo de servicio y las pruebas se llevaron a cabo con los parámetros de la tabla 6 .

En la figura 4 se muestra $L_{S}$ para cada prueba de $C V_{T_{S}}^{2}$, como es de esperarse, al aparecer un cuello de botella el valor crece de manera considerable en comparación con las pruebas con servicio uniforme y, más aún, si en dicho cuello de botella aumenta la variación, también aumenta $L_{S}$.

A modo de ejemplo, si por alguna circunstancia aparece un cuello de botella y suponiendo que el tiempo de servicio sigue siendo de tipo exponencial $\left(C V_{T_{S}}^{2}=1\right)$, entonces el valor de $L_{S}$ cambia de 5.37 a 7.03 de acuerdo con el modelo analítico, en otras palabras, hay un incremento de $30 \%$ (tabla 7); más aún, si dicho cuello de botella además presenta una variación muy alta, por ejemplo $C V_{T_{S}}^{2}=1.5$, entonces $L_{S}$ aumenta a 7.33 , o bien, $36.49 \%$.

Tabla 4. Número de clientes en el sistema

\begin{tabular}{cccc}
\hline \multicolumn{4}{c}{$L_{S}$} \\
\hline$C V_{T_{S}}^{2}$ & Analítico & Simulado & Diferencia \\
\hline 1.5 & 6.15 & 6.98 & $-11.9 \%$ \\
1.25 & 5.81 & 6.23 & $-6.7 \%$ \\
1 & 5.37 & 5.42 & $-0.9 \%$ \\
0.85 & 5.25 & 5.07 & $3.6 \%$ \\
0.75 & 5.09 & 4.88 & $4.3 \%$ \\
\hline
\end{tabular}

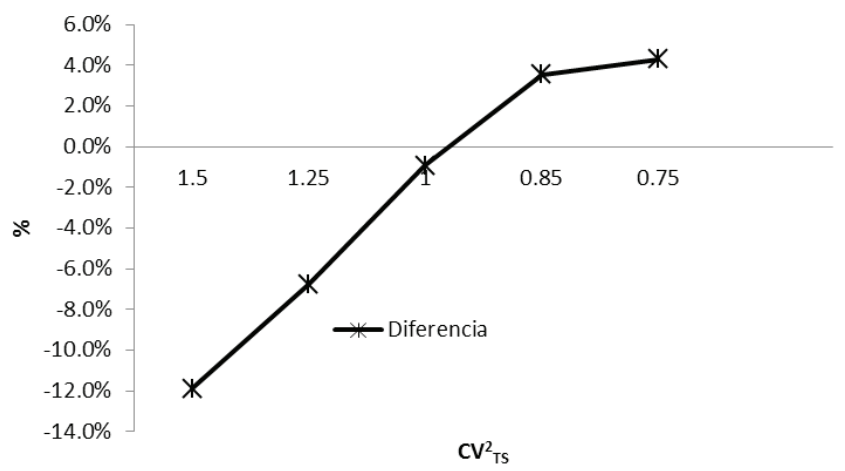

Figura 3. Diferencia vs $C V_{T_{S}}^{2}$

Tabla 5. Resultados de $p_{K}$ y $p_{0}$

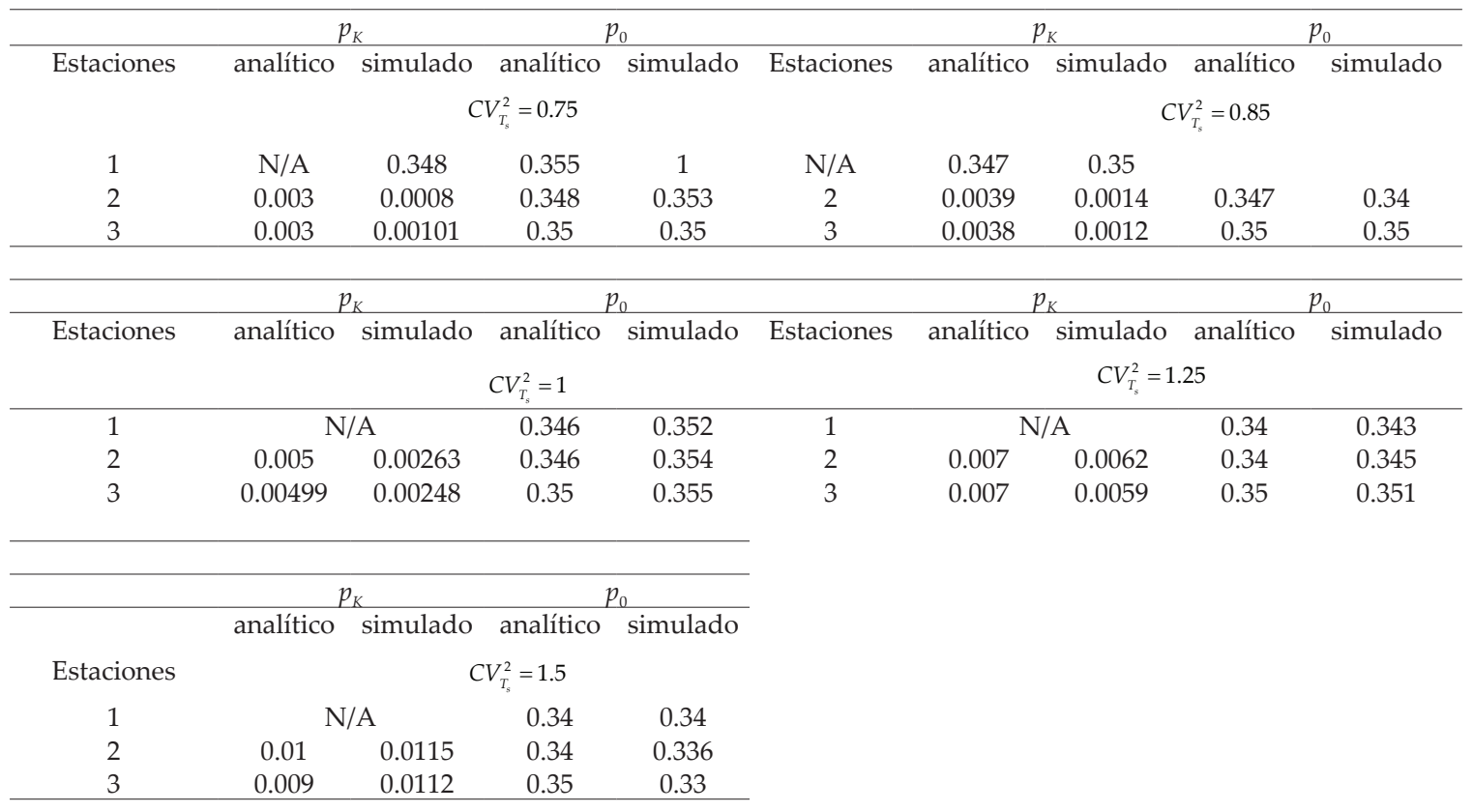


Tabla 6. Parámetros de la función gamma, valor esperado y varianza del cuello de botella

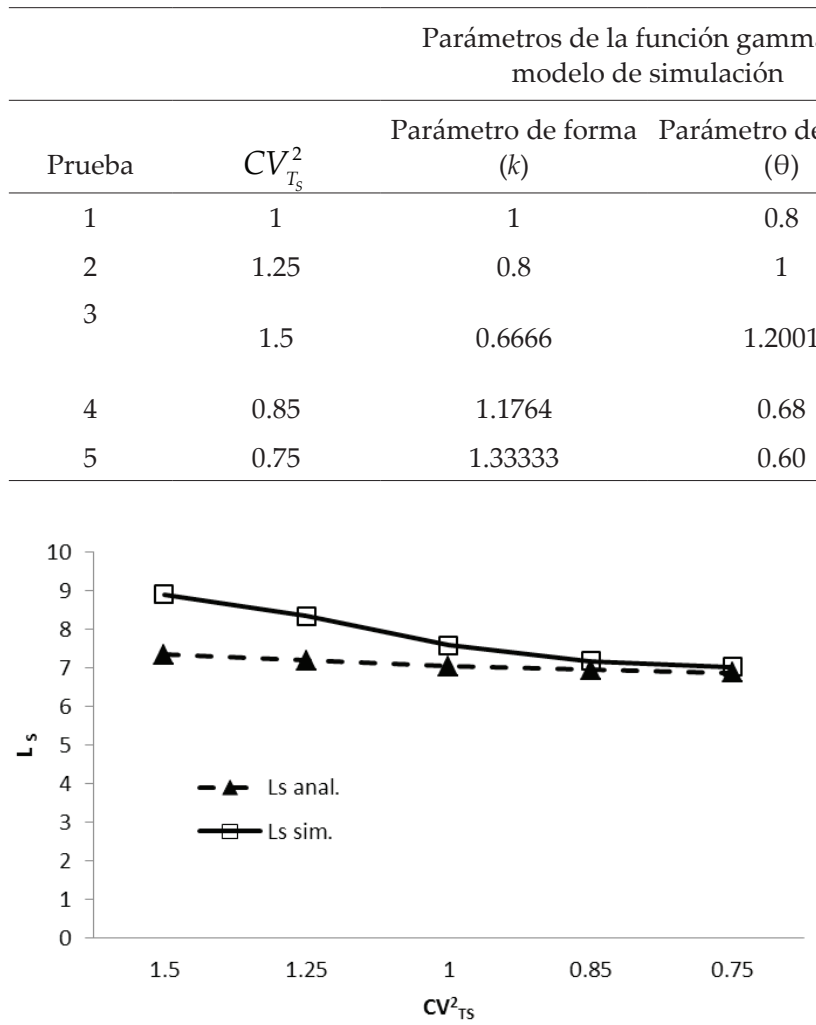

Figura 4. Número de clientes en el sistema vs $C V_{T_{S}}^{2}$. Cuello de botella en la estación 2

Tabla 7. Número de clientes en el sistema con cuello de botella

\begin{tabular}{cccc}
\hline \multicolumn{3}{c}{ Ls } \\
\hline$C V_{T_{S}}^{2}$ & Analítico & Simulado & Diferencia \\
\hline 1.5 & 7.33 & 8.89 & $-17.5 \%$ \\
1.25 & 7.18 & 8.33 & $-13.8 \%$ \\
1 & 7.03 & 7.58 & $-7.3 \%$ \\
0.85 & 6.94 & 7.17 & $-3.2 \%$ \\
0.75 & 6.86 & 7.01 & $-2.1 \%$ \\
\hline
\end{tabular}

También en la figura 4 se observa que $L_{S}$ obtenido de manera analítica se ve afectado a medida que se incrementa la variación del sistema (figura 4 y tabla 7).

En la figura 5 se observa graficada la diferencia de $L_{S}$ obtenida con el modelo analítico, y la obtenida mediante simulación, el comportamiento es el mismo que se obtuvo en la serie de pruebas anterior: si $C V_{T_{S}}^{2}$ disminuye, mejora la exactitud del modelo analítico.

En la figura 6 se muestra el número de clientes formados frente al cuello de botella (estación 2) y se obser-
Datos para el modelo analítico

\begin{tabular}{cc}
$\begin{array}{c}\text { Valor esperado del tiempo } \\
\text { de servicio } k \times \theta\end{array}$ & $\begin{array}{c}\text { Varianza } \\
k \times \theta^{2}\end{array}$ \\
0.8 & 0.64 \\
0.8 & 0.8 \\
0.8 & 0.96 \\
0.8 & 0.544 \\
0.8 & 0.48 \\
\hline
\end{tabular}

Figura 5. Diferencia vs $C V_{T_{S}}^{2}$ con cuello de botella

va que este disminuye a medida que el $C V_{T_{S}}^{2}$ disminuye también. En la operación, una disminución de la variación puede implicar, por ejemplo, que el servicio se realiza con mayor uniformidad entre cliente y cliente. También se aprecia que a medida que disminuye el valor de $C V_{T_{S}}^{2}$, el valor aproximado por el modelo analítico mejora.

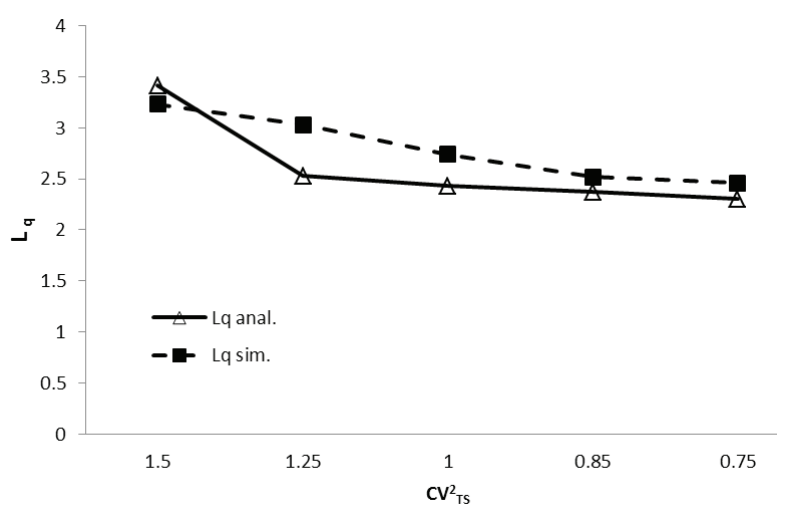

Figura 6. Número de clientes en la estación 2 (cuello de botella) vs $C V_{T_{S}}^{2}$ 
Tabla 8. Resultados de $p_{K}$ y $p_{0}$ con cuello de botella

\begin{tabular}{|c|c|c|c|c|c|c|c|c|c|}
\hline & & $p_{K}$ & & $p_{0}$ & & \multicolumn{2}{|c|}{$p_{K}$} & \multicolumn{2}{|c|}{$p_{0}$} \\
\hline Estación & & & \multirow{2}{*}{$\begin{array}{c}C V_{T_{s}}^{2}=0.75 \\
0.33\end{array}$} & \multicolumn{2}{|r|}{ Estación } & & \multirow[b]{2}{*}{0.337} & & \\
\hline 1 & & & & 0.337 & 1 & 0.329 & & & \\
\hline 2 & 0.021 & 0.019 & 0.19 & 0.2 & 2 & 0.0249 & 0.021 & 0.19 & 0.2 \\
\hline 3 & 0.0049 & 0.0012 & 0.35 & 0.35 & 3 & 0.0049 & 0.0016 & 0.35 & 0.35 \\
\hline & \multicolumn{2}{|c|}{$p_{K}$} & \multicolumn{2}{|c|}{$p_{0}$} & & \multicolumn{2}{|c|}{$p_{K}$} & \multicolumn{2}{|c|}{$p_{0}$} \\
\hline Estación & & & $C V_{T_{s}}^{2}=1$ & & Estación & & & $=1.25$ & \\
\hline 1 & & & 0.32 & 0.32 & 1 & & & 0.31 & 0.31 \\
\hline 2 & 0.029 & 0.028 & 0.19 & 0.2 & 2 & 0.04 & 0.038 & 0.19 & 0.196 \\
\hline \multirow[t]{2}{*}{3} & 0.0049 & 0.002 & 0.35 & 0.35 & 3 & 0.003 & 0.0049 & 0.35 & 0.35 \\
\hline & \multicolumn{2}{|c|}{$p_{K}$} & \multicolumn{2}{|c|}{$p_{0}$} & & & & & \\
\hline Estación & & & $C V_{T_{s}}^{2}=1.5$ & & & & & & \\
\hline 1 & N/A & 0.31 & 0.3 & & & & & & \\
\hline 2 & 0.047 & 0.049 & 0.19 & 0.19 & & & & & \\
\hline 3 & 0.0049 & 0.0044 & 0.35 & 0.35 & & & & & \\
\hline
\end{tabular}

El modelo analítico predice con bastante exactitud la probabilidad de bloqueo $\left(p_{K}\right)$ de la estación 2 (cuello de botella) para todas las pruebas de $C V_{T_{S}}^{2}$ (tabla 8). Para el caso de la estación 3 , se presenta la misma situación que en las pruebas con servicio uniforme: cuando $C V_{T_{S}}^{2}>1$ la diferencia con respecto al valor obtenido vía simulación disminuye.

\section{Conclusiones}

En este trabajo se presenta una adecuación al método de Buzacott que permite calcular de manera analítica las propiedades de sistemas de líneas de espera $M / G / 1 / K$ con arreglo en serie y los resultado se validaron mediante simulación.

El método tiene un grado de exactitud aceptable cuando se desea aproximar las propiedades de sistemas $M / G / 1 / K$ con arreglo en serie, congestión uniforme en las estaciones y valores del coeficiente de variación moderado. El valor de $p_{0}$ calculado es similar al obtenido mediante simulación, en el caso de $p_{K}$ la exactitud mejora a medida que el valor de $C V_{T_{S}}^{2}$ aumenta.

Para el caso donde existe cuello de botella, los resultados indican que el método es efectivo para calcular $L_{S}$ cuando la variación del cuello de botella es moderada $\left(C V_{T_{s}}^{2} \leq 1.25\right)$; a medida que la variación en el cuello de botella se incrementa más allá de este valor, la aproximación de $L_{S}$ se deteriora y por lo tanto los resultados deberán tomarse con reserva, en cambio, el valor de $p_{K}$ del cuello de botella se obtiene con un alto grado de exactitud.
La adecuación propuesta en este trabajo permite calcular las propiedades de sistemas $M / G / 1 / K$ con estaciones en serie. El grado de exactitud se ve afectado por $C V_{T_{S}}^{2}$, para valores bajos la exactitud de las ecuaciones mejora; por otro lado, el método tiene la ventaja de que su implementación en hoja de cálculo es rápida y sencilla.

El trabajo futuro puede encaminarse a estudiar la exactitud en sistemas con servidores múltiples, o bien, mejorar la plantilla en hoja de cálculo, por ejemplo para realizar cálculos dirigidos a optimización, sacando provecho de los complementos y macros disponibles o resolver instancias del problema de la localización del buffer (BAP), el cual hasta ahora se resuelve empleando técnicas heurísticas y metaheurísticas.

\section{Referencias}

Altiok T. Performance analysis of manufacturing systems, Nueva York, Springer, 1996.

Altiok T. y Melamed B. Simulation modeling and analysis with Arena, Oxford, Academic Press, 2007.

Buzacott J. y Shanthikumar G. Stochastic models of manufacturing systems, Upper Saddle River, Prentice Hall, 1993.

Cruz F.R. y MacGregor S.J. Approximate analysis of M/G/c/c state dependent queuing systems. Computers \& Operations Research, volumen 34 (número 8), agosto de 2007: 2332-2344.

Cruz F., Duarte A., Van-Woensel T. Buffer allocation in general single server queueing networks. Computers \& Operations Research, volumen 35 (número 11), noviembre de 2008: 3581-3598.

Curry G.L. y Feldman R.M. Manufacturing systems. Modelling and analysis, Berlín, Springer, 2011. 
MacGregor S.J. y Daskalaki S. Buffer allocation in automated assembly lines. Operations Research, volumen 36 (número 2), marzo-abril de 1998: 343-358.

Gelenbe E. On approximate computer system models. Journal of the Association for Computing Machinery, volumen 22 (número 2), abril de 1975: 261-269.

Gershwin S. An efficient decomposition method for approximate evaluation of tandem queues with finite storage space and blocking. Operations Research, volumen 35 (número 2), marzoabril de 1987: 291-305.

Hillier F.S. y Boling R.W. The effect of some design factors on the efficiency of production lines with variable operations times. Journal of Industrial Engineering, volumen 7, 1967: 651-658.

Kerbache L. y MacGregor S.J. The generalized expansion method for open finite queueing nteworks. European Journal of Operational Research, volumen 32, diciembre de 1987: 448-461.

Kimura T. A Transform-Free Approximation for the Finite Capacity M/G/s Queue. Operations Research, volumen 44 (número 6), noviembre-diciembre de 1996: 984-988.

Lawrence S.R. y Buss A. H. Economic analysis of production bottlenecks. Mathematical Problems in Engineering, volumen 1, febrero de 1996: 341-365.

MacGregor S.J. Optimal design and performance modelling of M/G/1/K queueing systems. Mathematical and Computer Modelling, volumen 39 (números 9-10), mayo de 2004: 1049-1081.

Perros H.G. y Altiok T. Approximate analysis of open networks of queues with blocking, Tandem configurations. IEEE transactions on software engineering, volumen 12 (número 3), marzo de 1986: 450-461.

Pollock S.M., Birge J.R., Alden J.M. Approximation analysis for open tandem queues with blocking: exponential and general service distributions. AnnArbor, 1985: 1-23.
Takahashi Y., Miyahara H., Hasegawa T. An approximation method for open restricted queueing networks. Operations Research, volumen 28 (número 3), mayo-junio de 1980: 594-602.

Tan B., Yeralan S. Analysis of multistation production systems with limited buffer capacity. Part 2: The decomposition method. Mathematical and Computer Modelling, volume 25 (número 11), junio de 1997: 109-123.

Van-Vuuren, M., Adan I., Resing-Sassen S. Performance analysis of multi-server tandem queues with finite buffers and blocking. OR Spectrum, volumen 27 (números 2-3), junio de 2005: 315-338.

Van-Woensel T., Andriansyah R., Cruz F.R., MacGregor S.J., Kerbache L. Buffer and server allocation in general multi-server queueing networks. International Transactions in Operational Research, volumen 17 (número 2), marzo de 2010: 257-286.

\section{Este artículo se cita:}

\section{Citación estilo Chicago}

Hernández-González, Salvador, Manuel Darío Hernández-Ripalda, Idalia Flores-De la Mota, José Antonio Vázquez-López. Propiedades de sistemas tipo $\mathrm{M} / \mathrm{G} / 1 / \mathrm{K}$ con estaciones en serie. Ingeniería Investigación y Tecnología, XVI, 03 (2015): 345-354.

\section{Citación estilo ISO 690}

Hernández-González S., Hernández-Ripalda M.D., Flores-De la Mota I., Vazquez-Lopez J.A. Propiedades de sistemas tipo M/G/1/K con estaciones en serie. Ingeniería Investigación y Tecnología, volumen XVI (número 3), julio-septiembre 2015: 345-354.

\section{Semblanzas de los autores}

Salvador Hernández-González. Es investigador de tiempo completo en el Departamento de Ingeniería Industrial del Instituto Tecnológico de Celaya. Tiene doctorado en investigación de operaciones por la Facultad de Ingeniería de la Universidad Nacional Autónoma de México. Su línea de investigación es la aplicación de modelos matemáticos para toma de decisiones.

Manuel Darío Hernández-Ripalda. Es profesor de tiempo completo en el Departamento de Ingeniería Industrial del Instituto Tecnológico de Celaya. Tiene la maestría en ciencias de la ingeniería (investigación de operaciones) por el Instituto Tecnológico de Estudios Superiores Monterrey. Su interés de investigación son la estadística y la probabilidad aplicada a la solución de problemas industriales.

Idalia Flores- De la Mota. Obtuvo el doctorado en investigación de operaciones que cursó en la Facultad de Ingeniería de la Universidad Nacional Autónoma de México. Sus intereses de investigación son programación entera, modelos de redes y simulación de sistemas industriales.

José Antonio Vázquez-López. Es investigador de tiempo completo en el Departamento de Ingeniería Industrial del Instituto Tecnológico de Celaya. Obtuvo el doctorado en ciencia y tecnología en ingeniería industrial por el PICyT-CIATEC, León. Sus intereses de investigación son la aplicación de redes neuronales para la solución de problemas de toma de decisiones en ambientes de producción. 